\title{
Schlussbetrachtung und Ausblick
}

Wie ,konstruiert' die deutsche Automobilindustrie ihr Wissen? Um das herauszufinden, hat die hier vorliegende Forschungsarbeit die organisationale Wissensgenese und Wissensverwendung anhand der angewandten Praxis der Methode der Social-Media-Analysen am Fallbeispiel eines deutschen Automobilherstellers explorativ untersucht. Um für die empirische Untersuchung einen theoretischen Bezugsrahmen abzustecken, wurde in einem ersten Schritt nicht nur Wissen als ein Gegenstand der Soziologie dargelegt, sondern auch Organisationen wurden als solcher thematisiert. Auf diese Weise ließ sich die wissenssoziologische Organisationsforschung als dasjenige Teilgebiet der Soziologie bestimmen, in dem die vorliegende Studie zu verorten ist. In diesem Zusammenhang wurde auch die wissenssoziologische Darlegung von Karl Mannheim (1929/1995) als relevant hinsichtlich der Ergebnisse der empirischen Untersuchung herausgestellt und die neue Wissenssoziologie nach Peter L. Berger und Thomas Luckmann (1966/2009) als der wissenssoziologische Theorierahmen der gesamten Forschungsarbeit - vor allem aber der empirische Untersuchung - vorgestellt. Innerhalb ihres theoretischen Teils hat diese Studie zudem in einem zweiten Schritt Social-Media-Analysen als eine innovative und digitale Methode der Wissensgenese, welche heute vor allem im Kontext der Computational Social Sciences und Digital Humanities zu positionieren ist, dargelegt und mit der klassischen Marktforschung kontrastiert. Damit war ein umfassender theoretischer Bezugsrahmen für die empirische Untersuchung abgesteckt. Sodann fiel der Blick auf das Untersuchungsmaterial und das methodische Vorgehen der empirischen Analyse. Letztere war zum einen durch ihren explorativen Charakter und zum anderen durch ihre Orientierung an der Grounded Theory Methodologie geprägt. Die Fragestellung, wie die deutsche Automobilindustrie ihr Wissen ,konstruiert', wurde dann in zwei Schritten vorläufig beantwortet. Zunächst konnte mit deskriptivem Fokus die in der deutschen Automobilindustrie angewandte Praxis von 
Social-Media-Analysen und deren Bestimmung von Social-Media-Analysen als Methode präsentiert werden. Drei Befunde waren auszumachen: Erstens zeichnet sich die von Seiten des betrachteten Unternehmens angewandte Praxis von Social-Media-Analysen durch Kompetenzmangel und auch ,blindes Agenturvertrauen' aus. Zweitens gewinnen die befragten Praktiker anhand der Methode zwar „quick Wins" via „quick Shots“, wobei bei jeder Social-Media-Analyse auch immer, weiße Flecken' bestehen bleiben. Drittens integriert der deutsche Automobilhersteller Social-Media-Analysen in sein „Puzzlespiel“ und „Mosaiklegen ". Nach Darlegung dieser drei Befunde hat sich die Schwerpunktsetzung der Ergebnispräsentation verschoben; die Deskription trat hinter die Deutung zurück. Für das betrachtete Unternehmen ließen sich zwei Typen der Wissensverwendung rekonstruieren: Einerseits die Orientierung, die durch die Exploration von Wissen entsteht; andererseits die Legitimation, die auf der Reflexion von Wissen beruht. Anschließend wurde die Bedeutung, die das Unternehmen Wissen beimisst und die spezifische Art der von ihm praktizierten Wissensgenese herausgearbeitet: Der deutsche Automobilhersteller begreift das über die Methode der Social-Media-Analysen generierte Netzöffentlichkeitswissen als Ressource; diese instrumentalisiert er ,innerhalb seiner Mauern'. Er ,konstruiert' diese Ressource durch affirmative Forschung, wobei seine Praxis der Social-Media-Analysen dem Prinzip „Muddling Through“ unterliegt. Eine explorative Untersuchung von Praktikerwissen, wie in dieser Studie realisiert, hat damit den organisationalen Konstruktionsprozess von Wissen in einem Unternehmen der deutschen Automobilindustrie erschlossen. Dieser beruht auf der Untersuchung von Netzöffentlichkeitswissen anhand der Methode der Social-Media-Analysen und erfolgt in Gestalt affirmativer Forschung; Letztere wird nach dem Prinzip „Muddling Through" umgesetzt.

Das untersuchte Praktikerwissen ist ebenso wie die rekonstruierte Wissensgenese und Wissensverwendung der Ideologie des deutschen Automobilherstellers unterworfen. Es handelt sich dabei um ein instrumentelles Ingenieursdenken mit ,quantitativer Logik' und Praktiker-Bias. Trotz dieser Ideologie waren auch utopische Momente ,innerhalb der Mauern“ des betrachteten Unternehmens auszumachen. Die Frage, welchen Umgang die Ideologen des deutschen Automobilherstellers mit dessen Utopisten pflegen und wie sie sich darum bemühen, dass ihre Ideologie nicht durch deren Utopie transformiert wird, hat diese Studie bislang allerdings offengelassen. Eine Antwort darauf enthält Bergers und Luckmanns Theorie der Wissenssoziologie. Sie spiegelt sich, bald mehr, bald weniger deutlich, bald nur latent und bald auch manifest in der von dem deutschen Automobilhersteller angewandten Praxis von Social-Media-Analysen wider. Damit ist diese Antwort ebenso in der Wissensgenese und Wissensverwendung 
des betrachteten Unternehmens sowie in der Wissenskonstruktion der deutschen Automobilindustrie zu erkennen. Es handelt sich um die Praktiken der „Therapie“ und der ,Nihilierung“"

Therapie bedient sich einer theoretischen Konzeption, um zu sichern, daß wirkliche oder potentielle Abweichler bei den institutionalisierten Wirklichkeitsbestimmungen bleiben. Sie soll mit anderen Worten verhindern, daß „Einwohner“ einer bestehenden Sinnwelt ,auswandern“. Zu diesem Zweck wendet sie den Legitimationsapparat auf individuelle „Fälle“ an. Da jede Gesellschaft mit der Gefahr individueller Abweichung rechnen muß, ist Therapie in dieser oder jener Form wahrscheinlich ein globales gesellschaftliches Phänomen (Berger, \& Luckmann, 1966/2009, S. 121).

Bei der Therapie handelt es sich, so erläutern Berger und Luckmann, um eine Kategorie sozialer Kontrolle. Als solche kontrolliere sie Abweichungen von den Bestimmungen der, Wirklichkeit', im Fallbeispiel dieser Studie also Abweichungen von der Ideologie des betrachteten Unternehmens, und stärke weiterhin die von diesen Abweichungen in Frage gestellte ,Wirklichkeit'. Laut Berger und Luckmann erschüttern Abweichungen, wie utopische Vorstellungen in Bezug auf die Schöpfung des explorativen Potentials von Social-Media-Analysen, inklusive einer Praxis des Deutens, oder womöglich sogar Äußerungen dieses utopischen Gedankenguts die gesellschaftliche ,Wirklichkeit', die bei dem betrachteten Unternehmen der deutschen Automobilindustrie in erster Linie auf instrumentellem Ingenieursdenken gründet, fundamental, da sie deren ,Gewissheit“ ins Wanken bringt:

Eine theoretische Konzeption wie diese ermöglicht nun die praktische Therapie geeigneter Spezialisten und kann auch von der Person, welche von der Abweichung betroffen ist, internalisiert werden. [. . . ] Unter der Last seiner Schuld ist das gequälte Individuum bald bereit, seinen Zustand subjektiv im Sinne der theoretischen Konzeption zu akzeptieren, so wie er ihm von den Praktikern der Therapie dargestellt wird. Er kommt zur „Einsicht“, das heißt, die Diagnose wird subjektiv wirklich für ihn (Berger, \& Luckmann, 1966/2009, S. 122).

Eine Therapie sei notwendig, um alle ,bei der Stange“ zu halten; im Fallbeispiel dieser Forschungsarbeit hieße das, alle Mitarbeiter des betrachteten Unternehmens innerhalb seiner „Sinnwelt“, die in seiner Ideologie besteht, zu ,verhaften“. 
Die zweite von Berger und Luckmann erkannte Praktik, die Nihilierung, geht mit der Therapie einher oder greift, wenn diese nicht fruchtet:

Nihilierung umgekehrt braucht sie, um alles, was außerhalb dieser Sinnwelt steht, mindestens theoretisch zu liquidieren. Man kann ein solches Verfahren auch eine Form von negativer Legitimation nennen. Nihilierung leugnet die Wirklichkeit von Phänomenen (beziehungsweise ihre Interpretationen), die nicht in die betreffende Sinnwelt hineinpassen (Berger, \& Luckmann, 1966/2009, S. 123).

Praktiziert werden kann die Nihilierung laut Berger und Luckmann auf zwei Weisen. Erstens ist es möglich, abseitige Phänomene mit einem negativen ontologischen Status zu belegen; dies kann mit oder auch ohne therapeutische Absicht vonstattengehen:

Mit der Nihilierung entsteht zweitens der Ehrgeiz, alle abweichenden Wirklichkeitsbestimmungen mit Begriffen aus der eigenen Sinnwelt angehen zu können. [. . . ] Die abweichenden Auffassungen werden nicht nur mit einem negativen Status versehen, sondern es wird im einzelnen theoretisch mit ihnen gerungen. Das Endziel dieses Vorgehens ist, sie der eigenen Sinnwelt einzuverleiben und so endgültig zu liquidieren. Deshalb müssen sie in Begriffe ,übersetzt“" werden, die von der eignen Sinnwelt abgeleitet sind. Ihre Nihilierung der bedrohten Sinnwelt wird auf diese Weise listig in eine Bestätigung umgemünzt (Berger, \& Luckmann, 1966/2009, S. 123 f., Hervorheb. i. O.).

Therapie und Nihilierung, wie von Berger und Luckmann diagnostiziert, sind die zwei Praktiken, die den Umgang der Ideologen des deutschen Automobilherstellers mit den Utopisten bestimmen, die sich in diesen ,hineinverirrt ' haben, ihre Existenz dort im Verborgenen fristen und es wagen, entsprechend ihrer Utopie zu handeln. Den Ideologen geht es darum, ihre Ideologie, welche die "Sinnwelt“" ihres Unternehmens ausmacht und dessen Denkstil bestimmt, zu wahren und Utopien, die dieser und damit der ,konstruierten ",Wirklichkeit" des deutschen Automobilherstellers widersprechen, vehement abzuwehren. Nihilierung bedeutet im Fallbeispiel dieser Studie, dass eine dem Unternehmen nicht vertraute Art von Forschung, eine, die explorativ vorgeht, als ein Widerspruch zu seiner eigenen, seit jeher etablierten Praxis, die in affirmativer Forschung besteht, ,verneint ${ }^{\text {* }}$ wird. Wie diese Studie gezeigt hat, ,depotentialisiert ${ }^{\circ}$ das betrachtete Unternehmen Social-Media-Analysen und delegitimiert damit die gesamte Methode, die ihr Potential in der Exploration entfalten könnte; dies geht mit einer Diskreditierung von Personen einher, die angelehnt an Berger und Luckmann, in Therapie oder Nihilierung bestehen kann. Berger und Luckmann beschreiben diese am Beispiel des „Intellektuellen“ oder auch „Experten“: 
Ein wichtiger Typ des Experten in der Geschichte [. . . ] ist der Intellektuelle. Wir neigen dazu, ihn als einen Experten zu bezeichnen, dessen Expertise von der Gesellschaft nicht gewünscht wird. Darin steckt, daß es ihm um eine Neubestimmung des Wissens im Angesichte seiner offiziellen Version zu tun ist, das heißt um mehr als nur eine abweichende Auslegung der letzteren. Der Intellektuelle wird damit per definitionem zu einem Grenzfall: er ist für die Gesellschaft marginal. [. . . ] In jedem Fall bringt seine gesellschaftliche Randexistenz einen Mangel an geistiger Integration in die Sinnwelt seiner Gesellschaft zum Ausdruck. Er wirkt als der Gegenexperte beim Handel mit der Bestimmung von Wirklichkeit. Wie der ,offizielle“ Experte hat er einen Entwurf für die ganze Gesellschaft. Aber während der Plan seines Kontrahenten mit den institutionellen Programmen übereinstimmt und diesen als theoretische Legitimation dient, schwebt der des Intellektuellen in einem institutionellen Vakuum, das gesellschaftlich noch am besten in einer Subgesellschaft intellektueller Leidensgenossen Gehalt annimmt. Wie weit solche Subgesellschaften lebensfähig sind, hängt von den strukturellen Verhältnissen in der größeren Gesellschaft ab (Berger, \& Luckmann, 1966/2009, S. 135).

Laut Berger und Luckmann bleiben dem Experten beziehungsweise Intellektuellen dann mehrere Optionen. Er kann sich in eine intellektuelle Subgesellschaft zurückziehen und bewahrt sich damit subjektiv seine abweichenden Konzeptionen, welche zwar von der größeren Gesellschaft nihiliert, von anderen Mitgliedern der Subgesellschaft aber als, Wirklichkeit' angesehen werden. In Bezug auf das Fallbeispiel dieser Arbeit bedeutet dies, dass sich die Utopisten des deutschen Automobilherstellers zwar zurückziehen, ihr utopisches Gedankengut aber im Verborgenen aufrechterhalten und mit Gleichgesinnten in Kontakt stehen. Zugleich sei der Intellektuelle darum bemüht, die ,Wirklichkeit' der Subgesellschaft zu bewahren; dies könne auch die Praktik der Therapie beinhalten. Entsprechend bewahren auch die Utopisten des betrachteten Unternehmens ihre Einschätzung des explorativen Potentials der Methode der Social-Media-Analysen und ,therapieren' sich in Bezug auf dieses gegenseitig; beispielsweise, wenn ein Utopist den Anschein erweckt, als würde er sich doch der etablierten Ideologie des instrumentellen Ingenieursdenkens unterwerfen. Eine alternative Option, die dem Intellektuellen nach Berger und Luckmann offensteht, ist die Revolution; dies meint, dass der Intellektuelle loszieht, um seine Konzeptionen zu verwirklichen. Eine solche Revolution war in dem betrachteten Unternehmen nicht auszumachen. In beiden Fällen ist eine Bestätigung durch Dritte vonnöten. Bisweilen werden ,revolutionäre Intellektuelle“ laut Berger und Luckmann auch zu ,offiziellen Legitimatoren“. Die „Legitimationsexperten“ können sowohl als Ideologen als auch als Utopisten, jeweils im Verständnis von Mannheim, agieren, denn „Wirklichkeitsbestimmungen“ haben - so die Annahme von Berger und Luckmann - die „Kraft der Selbstverwirklichung“": 
Sozialer Wandel muß also immer in dialektischer Beziehung zur Ideengeschichte gesehen werden. [ . . ] Eben diese Dialektik bewirkt, daß symbolische Sinnwelten sich wandeln. Soziologisch wesentlich ist, daß jede symbolische Sinnwelt und jede Legitimation Produkt des Menschen ist. Die Grundlage ihres Daseins ist das Leben lebendiger Menschen. Abgetrennt von dieser ihrer Grundlage besitzen sie keinen empirischen Status (Berger, \& Luckmann, 1966/2009, S. 137 f.).

Damit ist an dieser Stelle die Frage nach dem Umgang der Ideologen des betrachteten Automobilherstellers mit seinen Utopisten anhand von Bergers und Luckmanns Theorie der Wissenssoziologie vorläufig beantwortet.

Innerhalb des betrachteten Unternehmens war eine Vielzahl angewandter Praktiken der Methode der Social-Media-Analysen auszumachen, auch bezogen auf andere Verfahren, es gab jedoch keinen Überblick über die gesamte Praxis der Social-MediaAnalysen. Wie etliche andere Phänomene der Digitalisierung birgt auch die Methode der Social-Media-Analysen nicht nur technologische, sondern ebenso soziale Herausforderungen. Wie die hier vorliegende Studie gezeigt hat, sind mehrere Desiderate an die deutsche Automobilindustrie zu stellen: (1) Es ist an der Zeit, das explorative Potential von Social-Media-Analysen anzuerkennen, auch, wenn es in einem Widerspruch zu ihrem Denkstil steht. Die Methode bietet Chancen für die organisationale Wissensgewinnung. Social-Media-Analysen können, vor allem wenn sie zum Zweck explorativer Forschung praktiziert werden, Orientierung geben, durch diese zum Handeln befähigen und dies wiederum legitimieren. (2) Für die deutsche Automobilindustrie ist es unabdingbar, die für die praktische Anwendung von SocialMedia-Analysen nötige Methodenkompetenz zu entwickeln oder auszubauen. Es handelt sich dabei um Verfahren der Computational Social Sciences und Digital Humanities, mit denen ein reflektierter Umgang zu pflegen ist. Methodenkompetenz zu fördern bedeutet für die deutsche Automobilindustrie, dass sie Social-MediaAnalysen selbst umsetzt und Agenturen wie Dienstleistern nicht ,blind vertraut'. Vielmehr sollten die Praktiker über eigene Methodenkompetenz verfügen. Zu diesem Zweck besteht eine Option in der interdisziplinären Zusammenarbeit. Es gilt des Weiteren, Social-Media-Analysen nicht in das Thema „Big Data“, hineinzupressen“. Social-Media-Analysen sind als eine Methode der Sozialwissenschaften, die sich der Verfahren der Informatik bedient, zu begreifen. (3) Damit dies gelingt, ist innerhalb der deutschen Automobilindustrie ,methodisches Change-Management nötig. Fortschritt scheint möglich, wenn man bei der ,Konstruktion“ von Wissen einmal etwas , anders ' macht und dabei gegebenenfalls dem eigenen Denkstil zuwider handelt. Es gilt das methodische ,Mindset' $\mathrm{zu}$ öffnen und auch die digitalen Methoden wie Social-Media-Analysen zu nutzen; neben quantitativen Untersuchungen sind qualitative anzustellen. Eine methodisch fundierte Forschung kann auch pragmatisch durchgeführt werden. (4) Damit eine Online-Forschung möglich wird 
und andere Untersuchungen um das Wissen von der Netzöffentlichkeit und auch über sie ergänzt werden können, sollten Social-Media-Analysen in das Methodenrepertoire der deutschen Automobilindustrie fest integriert werden. Es geht dabei nicht darum, die etablierte Forschung und ihre Methoden durch Social-Media-Analysen zu ersetzen. (5) Eine Integration in das Methodenrepertoire der deutschen Automobilindustrie beinhaltet, dass Erkenntnisse, die über Social-Media-Analysen generiert wurden, mit anderen Forschungsresultaten verknüpft und ferner all denjenigen, für die diese Erkenntnisse von Bedeutung sein können, zugänglich gemacht werden. Um dies in der deutschen Automobilindustrie zu realisieren, bedarf es eines kontinuierlichen, in der Unternehmenspraxis fest etablierten Wissensmanagements, das sich über die einzelnen Organisationseinheiten erstreckt und diese im Hinblick auf ihr Wissen zu einer Einheit werden lässt, vonnöten. (6) Es scheint erforderlich, in Bezug auf die Forschung im Allgemeinen und Social-Media-Analysen im Speziellen und damit wiederum hinsichtlich der organisationalen Wissensgewinnung das etablierte Denken in organisationalen Einheiten aufzubrechen. Praktiker sollten sich untereinander vernetzen, möglicherweise im Rahmen einer, Task Force'. Das Ziel in der Unternehmenspraxis sollte darin bestehen, von dem Wissen der anderen zu profitieren und sich gemeinsam der Potentiale und Herausforderungen, der Chancen und Risiken, die mit Social-Media-Analysen einhergehen, bewusst zu werden und das explorative Erkenntnispotential zu nutzen, das die Methode bereithält.

In dieser Arbeit ist die Rede von der ,,deutschen Automobilindustrie“. Untersucht wurde die angewandte Praxis von Social-Media-Analysen am Fallbeispiel eines deutschen Automobilherstellers. Von diesem wurde auf die gesamte deutsche Automobilindustrie geschlossen. Auch wenn es bisweilen im öffentlichen Diskurs, zur Zeit der Entstehung dieser Studie im Kontext von „Dieselskandal“ und „Abgasaffäre", so wirkt als gleiche ein Automobilhersteller dem anderen und als würden alle gemeinsam ein „Kartell“ formieren, so scheint gerade im Hinblick auf die organisationale Wissensgenese und Wissensverwendung oder ,Konstruktion " von Wissen ein Vergleich zwischen mehreren Automobilherstellern, deutschen und internationalen, interessant. Des Weiteren könnte es bereichernd sein, diesen Vergleich mit Organisationen anderer Größenordnung zu ziehen, wie mit kleinen und mittleren Unternehmen (KMU) die der Automobilindustrie im weiteren Sinne angehören, oder auch mit anderen Industrien. Unzählige Varianten des Vergleichs sind vorstellbar und jede einzelne davon würde zu einem vollständigeren Bild beitragen, als es diese Studie allein zu zeichnen vermag (Dohmen, \& Hawranek, 2017).

Für die Verfasserin der vorliegenden Studie scheint jedoch eine Wiederholung oder eine Neuaufnahme der explorativen Untersuchung der ,Konstruktion“ von Wissen innerhalb des betrachteten Unternehmens am vielversprechendsten. Wenn ein paar Jahre seit der Veröffentlichung dieser Arbeit vergangen sein werden, 
deren Erkenntnisse mehrfach in dem betrachteten Unternehmen der deutschen Automobilindustrie präsentiert wurden und vor allem nachdem vermutlich der digitale Wandel, der unsere Gegenwart prägt und im öffentlichen Diskurs als das zeitdiagnostische Phänomen der „Digitalisierung“ gehandelt wird, fortgeschritten ist, sollte eine solche erneut durchgeführt werden. Dazu, ob sich mit der Zeit auch die Ideologie des deutschen Automobilherstellers und damit zugleich dessen Konstruktionsweise von Wissen verändern oder, ob sich stattdessen die Utopie der sozialwissenschaftlich reflektierten Forschung behaupten wird, die sich an explorative Methoden und deren Erkenntnispotential heranwagt, kann die Verfasserin derzeit selbst nur Hypothesen aufstellen. Da es sich hier jedoch nach wie vor um eine sozialwissenschaftliche Forschungsarbeit handelt, die für sich beansprucht, kritisch reflektiert entstanden zu sein, wird diese nicht mit subjektiven Mutmaßungen schließen, die einer objektiven Fundierung entbehren.

Für die Verfasserin dieser Forschungsarbeit war es eine Herausforderung, für ihre Untersuchung zugleich aber ein Potential, parallel und manchmal gleichzeitig die angewandte Praxis von Social-Media-Analysen in der deutschen Automobilindustrie $\mathrm{zu}$ erforschen und selbst in diese Praxis der Wissensgenese und Wissensverwendung des betrachteten Unternehmens eingebunden zu sein. Hier galt es stets eine professionelle Distanz zu wahren; mal zur Wissenschaft, mal zur Wirtschaft. Zudem war es essentiell, das eigene Handeln wiederholt aus einer Metaperspektive zu betrachten und überaus selbstkritisch zu reflektieren. Im Zuge dieses immerwährenden Prozesses kritischer Reflexion ist ein Dilemma zutage getreten. Es besteht darin, dass die Verfasserin zum einen selbst eine explorative Untersuchung umgesetzt, Erkenntnisse also durch eine Rekonstruktion, über Deutungen und Interpretationen gewonnen hat, und zum anderen aber anhand dieses explorativen Vorgehens herausgefunden hat, dass das betrachtete Unternehmen der deutschen Automobilindustrie das Potential explorativer Forschung derzeit verkennt. Es ist daher festzustellen, dass die Verfasserin der vorliegenden Arbeit in Bezug auf die anwendenden Praktiker des betrachteten Unternehmens eher den Utopisten als den Ideologen zuzurechnen ist; vielleicht am ehesten sogar den Experten oder auch Intellektuellen. Therapieversuche hat der deutsche Automobilhersteller ihr gegenüber während des gesamten Forschungsprozesses unternommen; geglückt sind diese nicht. Daher hat eine Nihilierung ihrer explorativen Untersuchung und deren Erkenntnisse stattgefunden. Eine Revolution hat daraufhin auch sie nicht begonnen. Wer weiß aber zum gegenwärtigen Zeitpunkt schon, wozu eine hiermit nun vorerst abgeschlossene Studie der Soziologie dann doch noch wachzurütteln vermag, denn ,Revolutionary Movements s sind dieser Disziplin bekanntlich eher nah als fern. 
Open Access Dieses Kapitel wird unter der Creative Commons Namensnennung 4.0 International Lizenz (http://creativecommons.org/licenses/by/4.0/deed.de) veröffentlicht, welche die Nutzung, Vervielfältigung, Bearbeitung, Verbreitung und Wiedergabe in jeglichem Medium und Format erlaubt, sofern Sie den/die ursprünglichen Autor(en) und die Quelle ordnungsgemäß nennen, einen Link zur Creative Commons Lizenz beifügen und angeben, ob Änderungen vorgenommen wurden.

Die in diesem Kapitel enthaltenen Bilder und sonstiges Drittmaterial unterliegen ebenfalls der genannten Creative Commons Lizenz, sofern sich aus der Abbildungslegende nichts anderes ergibt. Sofern das betreffende Material nicht unter der genannten Creative Commons Lizenz steht und die betreffende Handlung nicht nach gesetzlichen Vorschriften erlaubt ist, ist für die oben aufgeführten Weiterverwendungen des Materials die Einwilligung des jeweiligen Rechteinhabers einzuholen.

(c) (9) 